\title{
PERCEPCIÓN DE LA POBLACIÓN SOBRE LA VACUNA CONTRA EL COVID-19: PERSPECTIVAS EN REPÚBLICA DOMINICANA, 2020
}

\section{Population's perception on the vaccine against COVID-19: Perspectives in the Dominican Republic 2020}

\author{
Vahid Nouri Kandanya, María Isabel Marte ${ }^{b}$, Elly Pichardo ${ }^{c}$
}

Recibido: 12 de abril, $2021 \bullet$ Aprobado: 28 de mayo, 2021

Cómo citar: Nouri Kandanya V, Marte MI, Pichardo E. Percepción de la población sobre la vacuna contra el COVID-19: perspectivas en República Dominicana, 2020. cysa [Internet]. 13 de octubre de 2021 [citado 15 de octubre de 2021];5(3):27-5. Disponible en: https://revistas.intec.edu.do/index.php/cisa/article/view/2310

\section{Resumen}

La enfermedad del COVID-19 fue declarada pandemia en marzo de 2020, causando complicaciones de salud graves e incluso la muerte, a la fecha, en más de dos millones de personas a nivel mundial. Hasta el momento no existe ningún medicamento eficaz contra el COVID-19 y la única esperanza para mitigar la pandemia será el uso masivo de la vacuna. En esta misma línea, en la última década se han formado movimientos contra la vacunación en el mundo, creando incertidumbre sobre la efectividad de las mismas. Por tal razón son importantes las estrategias de educación sobre vacunación como pilares para el avance en políticas de salud pública en nuestro país.

Con el objetivo de determinar la percepción de la población en República Dominicana (R.D.) acerca de la vacunación contra el COVID-19, se diseñó un estudio descriptivo de corte transversal, usando un formulario en línea, compuesto por preguntas cerradas y semicerradas.

De un total de 841 respuestas válidas $(59.5 \%$ mujeres y $40.5 \%$ hombres) con una media de 31,33 años. La mayoría de los participantes $(43.3 \%, \mathrm{n}=364)$ fueron

\footnotetext{
a Centro de Investigación Biomédica (CINBIOCLI), Unidad de investigación Universidad Autónoma de Santo Domingo, Santiago de los Caballeros, República Dominicana. ORCID: 0000-0001-6361-5529

Correo-e: vahidkandany@gmail.com
}

\begin{abstract}
The COVID-19 disease was declared a pandemic in March 2020, causing severe health complications and deaths to date in more than two million people globally. So far, there is no effective drug against COVID-19. The only hope to mitigate the pandemic will be the massive use of the vaccine over the past decade; anti-vaccination movements have formed around the world, creating doubts about its effectiveness and other aspects. For this reason, vaccination strategies are essential pillars for the advancement of public health policies in our country.

To determine the perception of the population in the Dominican Republic about vaccination against COVID-19, a descriptive cross-sectional study was designed, using an online form composed of closed and semi-closed questions.

From a total of 841 effective responses $(59.5 \%$ women and $40.5 \%$ men) with a mean age of 31.33 years. Most of

\footnotetext{
b Vicerrectoría de Investigación, Universidad Tecnológica de Santiago (UTESA). Santiago de los Caballeros, República Dominicana. ORCID:0000-0002-5262-8989, Correo-e: isabelmarte@hotmail.com

c Facultad de Ciencias de la Salud, Pontificia Universidad Católica Madre y Maestra (PUCMM). Santiago de los Caballeros, República Dominicana. ORCID:0000-0002-8228-6393
}

Correo-e: eapichardo6@gmail.com
\end{abstract}


residentes de la provincia de Santiago de los Caballeros $(\mathrm{n}=364 ; 43.3 \%)$. Los estudiantes universitarios representaron el $45.1 \%(n=379)$ de la población encuestada. Actualmente, 208 de los participantes están desempleados $(24.7 \%)$, la comorbilidad más frecuente fue la hipertensión arterial (11.4\%). Un $44.7 \%$ de ellos respondió positivamente a la eventual aplicación de la vacuna y el género con mayor aceptación fue el masculino (54.3\%), solo un $28.8 \%$ contestó que se vacunará en cuanto haya disponibilidad; $61.6 \%$ piensa que la vacuna será moderadamente efectiva, $26.2 \%$ temen de los efectos adversos en futuro y un $11.2 \%$ cambiaría su opinión si una figura pública se aplicase la vacuna. La información por parte de las autoridades locales e internacionales no tuvo alcance para un $70.4 \%$ de los participantes. Un $68 \%$ de la población tiene dudas sobre el desarrollo de la vacuna y un $70.7 \%$ considera que no debe ser obligatoria su aplicación. La mayoría confía en el trabajo de los científicos $(68.5 \%)$ y en las empresas farmacéuticas $(58.4 \%)$ acerca del desarrollo de la vacuna.

La población de estudio mostró una percepción no favorable hacia la vacunación contra COVID-19, evidenciando la necesidad de implementar una campaña de información y orientación masiva.

Palabras clave: vacuna; COVID-19; Organización Mundial de la Salud; República Dominicana; percepción.

\section{Introducción}

El SARS-CoV-2, el virus que provoca la enfermedad conocida como COVID-19, se detectó por primera vez en seres humanos en Wuhan, China, a finales del 2019. Una neumonía de causa desconocida y que fue declarada brote y una "Emergencia de salud pública" por la Organización Mundial de la Salud; luego, como pandemia el 11 de febrero de $2020{ }^{1}$

Un año después se han registrado más de 90 millones de casos, 1,9 millones de fallecimientos y alrededor de 50,1 millones de personas recuperadas. ${ }^{2}$ Las autoridades del Ministerio de Salud Pública de República Dominicana confirmaron el primer caso de COVID-19 en la provincia La Altagracia el 1 de marzo de 2020, tratándose de un masculino de 62 años, quien ingresó al país el 22 de febrero de 2020 , the participants were residents of Santiago de los Caballeros $(43.3 \%, n=364)$. College students represented $45.1 \%(n=379)$ of the population. Currently, 208 of the participants are unemployed $(24.7 \%)$. The most frequent comorbidity was arterial hypertension (11.4\%). Of those surveyed, $44.7 \%$ answered affirmatively to the vaccine's application, and the gender with the highest acceptance was male $(54.3 \%)$. As soon as the vaccine becomes available, $28.8 \%$ answered that they would get vaccinated, $61.6 \%$ think that the vaccine would be moderately effective, and $26.2 \%$ fear adverse effects in the future. $11.2 \%$ would change their opinion if a public figure applied the vaccine. The information from the authorities did not reach $70.4 \%$ of the participants. $68 \%$ of the population have doubts about the development of the vaccine, and $70.7 \%$ consider that its application should not be mandatory. Most trust the work of scientists $(68.5 \%)$ and pharmaceutical companies $(58.4 \%)$ regarding the vaccine's development.

The study population showed an unfavorable perception of vaccination against COVID-19, which required massive information and an orientation campaign.

Keywords: Vaccine; COVID-19; World Health Organization; Dominican Republic; perception.

procedente de Italia. ${ }^{3}$ Desde entonces, hasta el 19 de diciembre, han fallecido 2,385 personas y se han registrado 161,052 infectados. $^{4}$

Las consecuencias de la propagación del COVID-19 a nivel mundial y los daños humanos y económicos obligan a los gobiernos a centrar su atención en la investigación, elaboración y desarrollo de la vacuna contra esta enfermedad ${ }^{5}$.

En el contexto histórico de las vacunas, estas han sido vistas como herramientas útiles contra enfermedades infecciosas ${ }^{6}$, sin embargo, el posmodernismo se cuestiona el papel legítimo de la ciencia, dando así origen a movimientos antivacunas; y como efecto colateral se ha observado una resistencia creciente contra la vacunación desde hace más de una década, 
convirtiéndose en un problema global para la salud pública. ${ }^{7-10}$

Entre los aspectos que pueden influir en el rechazo a la aplicación de vacunas están: la historia social, cultural, política, las experiencias con los servicios de salud, antecedentes familiares, e incluso, opinión de los amigos cercanos; lo que obliga a contemplar estos aspectos desde un contexto más amplio.

"La cultura local sobre la vacunación" fue un término utilizado por Streefland et al. para conceptualizar la percepción de la población sobre el origen de las enfermedades, la idea sobre eficacia de la medicina moderna, tradicional, las medidas preventivas y la opinión de la población sobre el servicio de salud recibido en su localidad influye en su decisión para vacunarse o vacunar a sus hijos. ${ }^{11-12}$

Múltiples líderes mundiales han hecho pública su opinión acerca de recibir la vacuna, por ejemplo, en Estados Unidos algunos expresidentes expusieron su voluntariedad a aplicarla tan pronto como esté aprobada por la $\mathrm{FDA}^{13}$, en pos de fomentar seguridad y confianza a la población. En República Dominicana, tanto el presidente, la vicepresidente y la directora del Gabinete de Salud, expresaron que serían los primeros en vacunarse cuando hubiese disponibilidad en la isla. ${ }^{14}$

En el historial de efectos adversos de algunas vacunas se pueden mencionar varios casos de rechazo a la vacunación, como la asociación entre la vacuna contra la hepatitis $\mathrm{B}$ y la esclerosis múltiple en Francia; de igual manera se reportó un caso de asociación entre la vacuna triple vírica y el autismo en Reino Unido. ${ }^{15,16}$

Es muy importante contar con una política de educación formativa que motive y oriente a la población, en vez de recurrir a una educación correctiva y de carácter obligatorio, evitándose así la resistencia de la población a las campañas de vacunación y motivando a la colaboración en su beneficio.
Este trabajo tiene como objetivo determinar los niveles de aceptación de la población sobre la vacuna contra el COVID-19 en la población de la República Dominicana, creando evidencia para sugerir políticas educativas desde el gobierno, específicamente el Ministerio de Salud Pública, con la intervención de organizaciones de la sociedad civil y medios de comunicación, incentivando de manera proactiva la cultura de vacunación en general y, debido a la situación actual, en especial la vacuna contra el COVID-19.

\section{Material y métodos}

Se realizó un estudio descriptivo, prospectivo, de corte transversal, en un período de recolección de siete días con el objetivo de determinar la percepción de la población sobre la vacuna contra el COVID-19 en República Dominicana.

Fueron incluidos todos los residentes permanentes o temporales en República Dominicana en el intervalo de recolección de datos, con una edad $\geq 18$ años, que aceptaran participar firmando de manera virtual el consentimiento informado. Aquellos participantes reclutados completaron un cuestionario que consistió en 20 preguntas cerradas y semicerradas.

Se recolectaron datos personales, características demográficas, educacionales y profesionales, estimado de ingreso mensual y preguntas dirigidas a evaluar la percepción sobre la vacuna contra el COVID-19.

El cuestionario fue alojado en el servicio de Google Docs, y fue distribuido a través de la aplicación WhatsApp.

Se envió el instrumento de estudio, usando el método de muestreo no probabilístico por conveniencia y de "bola de nieve".

Los resultados obtenidos se tabularon en Excel 2020 y fueron analizados en SPSS 26, cuyo análisis 
estadístico descriptivo se basó en la media y desviación estándar para la variable continua de edad y la frecuencia absoluta para variables categóricas.

\section{Resultados}

De un total de 926 personas, $869(93.8 \%)$ respondieron afirmativamente a participar en el estudio $y$, de estos, 841 cumplieron con criterios de inclusión para una efectividad de $90.32 \%$ (véase tabla 1).

La edad de los participantes estuvo comprendida en un rango de 18-74 ańos, con una media de $31,33 \pm 11,22$ y una moda de 25 ańos.

La distribución de los participantes en el territorio nacional fue principalmente en las siguientes provincias: Santiago de los Caballeros ( $\mathrm{n}=364 ; 43.3 \%$ ), Distrito Nacional ( $\mathrm{n}=153 ; 18.2 \%)$, Santo Domingo $(\mathrm{n}=67 ; 8 \%)$, Puerto Plata ( $\mathrm{n}=54 ; 6.4 \%)$, Espaillat $(\mathrm{n}=39 ; 4.6 \%)$. Otras contaron con una participación menor de un por ciento cada uno, como, por ejemplo: Elías Piña, Azua, Bahoruco, Independencia, Peravia y Hato Mayor.

Respecto al mayor nivel educativo alcanzado, el $45.1 \%(\mathrm{n}=379)$ son estudiantes universitarios, mientras que un $28.3 \%(n=238)$ ha alcanzado un nivel de grado (ingenieros, licenciados y doctores en Medicina), $20.9 \%(\mathrm{n}=176)$ tiene nivel de maestría y el resto se divide entre personas no escolarizadas, técnicos, nivel de $\mathrm{PhD}$, así como nivel primario y secundario. Un $34.4 \%(n=289)$ de la población de estudio son profesionales de la salud.

La mayoría de las personas encuestadas $(\mathrm{n}=208$, $24.7 \%$ ) no percibe ingresos económicos, seguido de un $18.7 \%(\mathrm{n}=157)$, que recibe un ingreso mayor a $\mathrm{RD} \$ 63,000$.

Entre los grupos de riesgo a los cuales pertenecen los participantes encontramos, en orden de frecuencia: hipertensión arterial $(11.4 \%)$, enfermedad pulmonar (7.4\%), diabetes (3.5\%), fumadores $(2 \%)$, embarazadas (1.5\%), mayor de 65 años (1.4\%), cáncer (1\%), VIH/SIDA (0.6\%).
De las 841 personas, $44.7 \%(n=376)$ respondieron afirmativamente a la eventual aplicación de la vacuna, $38.3 \%$ no está seguro/a $(n=322)$ y el $17 \%$ restante $(\mathrm{n}=143)$ respondió negativamente. La respuesta positiva para la vacunación fue más alta entre los hombres (54.3\%) que en las mujeres $(38.2 \%)$.

Respecto al contexto temporal al cual los participantes estarían de acuerdo de aplicarse la vacuna, vemos en orden de frecuencia que: $28.8 \%(\mathrm{n}=242)$ lo haría en cuanto esté disponible, $14.7 \%(n=124)$ esperaría entre 6 meses a 1 año, $14 \%(\mathrm{n}=118)$ esperaría de 3 a 6 meses, $13.4 \%(n=113)$ esperaría de 1 a 2 meses, $12 \%(n=101)$ esperaría más de 1 año. En cuanto a los géneros, la tendencia de los hombres apunta a que se vacunarían inmediatamente esté disponible la vacuna con un $36.4 \%$ respecto al $23.6 \%$ de las mujeres.

Más de la mitad de la población del estudio considera que la vacuna será moderadamente efectiva $61.6 \%$ $(\mathrm{n}=158), 17 \%(\mathrm{n}=143)$ cree que será totalmente efectiva, $16.4 \%(\mathrm{n}=138)$ será poco efectiva y un $5 \%(\mathrm{n}=42)$ nula efectividad. Los mayores temores de la población para vacunarse fueron: desarrollo de enfermedades secundarias en el futuro (26.2 \%), efectos adversos serios e inmediatos (19.4\%) y $22 \%$ expresó no tener ningún temor a vacunarse.

El hecho de que una figura pública de la admiración de los encuestados se aplicase la vacuna influiría positivamente en la decisión de aplicarla en un $11.2 \%(n=94)$.

Respecto a la información suministrada por parte del Ministerio de Salud Pública de la República Dominicana o la OMS acerca de la vacuna contra el COVID-19, $70.4 \%(\mathrm{n}=592)$ respondió no haberla recibido. Un $68.5 \%$ admite confiar en la comunidad científica que trabaja para empresas farmacéuticas para desarrollar la vacuna contra el COVID-19. De igual manera, 491 (58.4 \%) confía en las casas farmacéuticas que fabrican dichas vacunas. 
La brevedad con la que se aprobó la vacuna contra el COVID- 19 causa dudas al $68 \%(\mathrm{n}=592)$ de los participantes. Del total de los encuestados, un 62.3 $\%(\mathrm{n}=524)$ estaría de acuerdo con que se le aplique la vacuna a sus familiares, hijos y amigos, mientras que el $37.7 \%(n=317)$ restante no lo está. Un $70.7 \%$ $(\mathrm{n}=595)$ piensa que la vacuna no debe aplicarse con carácter de obligatoriedad.

\section{Discusión}

Los resultados derivados de este estudio muestran la percepción de la población dominicana sobre la vacuna contra el COVID-19. Dicha encuesta fue distribuida a finales de diciembre de 2020.

En el comienzo de la pandemia se realizó un estudio sobre aceptación de la vacuna contra el COVID-19 en 19 países del mundo, en una población que representa alrededor del $55 \%$ de la población mundial, y en el cual los encuestados de China dieron la mayor proporción de respuestas positivas (88.6\%), los rusos dieron la proporción más baja de respuestas positivas $(54.9 \%)$, mientras que los encuestados de Polonia representaron la mayor proporción de respuestas negativas $(27.3 \%)$. En dicho estudio la aceptación de la vacuna a nivel global fue de $46.8 \%$, muy similar a nuestro país (44.7\%); al contrario, en Ecuador, un país con similares características al nuestro, fue de $71.93 \%$, probablemente por la diferencia en fecha de encuesta. ${ }^{17}$

Los resultados del presente trabajo demuestran que un pequeño porcentaje de la población no se aplicará la vacuna $(17 \%)$, y entre las personas que están de acuerdo con vacunarse existe un gran escepticismo, dudas al respecto, quienes dicen esperarían a ver los resultados en otras personas antes de usar la vacuna $(71,2 \%)$ casi dos veces más de estudio realizado en Espańa en julio de 2020. ${ }^{18}$

La participación de las mujeres fue más notable $(59.5 \%)$, pero con mayores dudas y menor aceptación que los hombres (80.8\% vs. $86.2 \%)$ que nos indica la necesidad de fortalecer la campaña de concientización hacia este grupo.

En cuanto a la edad de los participantes la mayoría tenían edades por debajo de 55 años (92.6\%), con mayor interés para vacunarse, contraria de un estudio encontrado en Estados Unidos ${ }^{19}$, esto sugiere mayor interés e información de este grupo de edad sobre el tema de vacunación.

Los participantes con nivel educativo más alto demostraron mayor aceptación (técnico $33.3 \%$ vs. licenciatura $35,4 \%$ vs. maestría $53,3 \%$ ), es preocupante un nivel de aceptación de $53.6 \%$ entre los trabajadores de salud en comparación del resto de población con un $40 \%$, constatando la necesidad de buscar estrategias especiales en dicho grupo, que son pilares importantes de promoción, prevención y la educación para la salud.

Según los resultados de este estudio, se revela la necesidad de enfatizar la campaña de orientación hacia las personas con mayor edad, con menores niveles educativos y sexo femenino, quienes demostraron menor aceptación para vacunarse.

Encontramos un elevado nivel de confianza en la población hacia la industria de fabricación de la vacuna $(58,4 \%)$ y los científicos que laboran en ella $(68,5 \%)$, pero notablemente se negó $(88,8 \%)$ el deseo de aplicación de la vacuna, aunque esta sea usada por una figura pública, siendo un dato relevante en la promoción en las campañas de vacunación.

La confianza en las vacunas se midió en 149 países del 2015 al 2019, en un estudio a nivel global, en el cual se evidenció una disminución de la confianza en la seguridad y la efectividad de las vacunas en las personas ${ }^{20}$, que coincide con resultados de nuestro estudio donde solo un $61.6 \%$ afirma que la vacuna contra el COVID-19 será moderadamente efectiva, $17 \%$ lo consideran totalmente efectiva, además generó muchas dudas la rapidez de comercialización de la vacuna $(68 \%)$ datos que nos ayudarán a 
fortalecer una campaña de vacunación dando suficiente información, mejorando la confianza de la población hacia la vacuna.

Los mayores temores de la población para vacunarse fueron: desarrollo de enfermedades secundarias en el futuro y efectos adversos inmediatos, algo menor en relación con una encuesta realizada en Estados Unidos con $47 \%, 22 \%$ vs. 26,2 \% y $19,4 \%$, respectivamente. ${ }^{21}$

El origen de estos temores probablemente sea resultado de escasez de información de parte de las autoridades locales e internacionales como afirman los encuestados $(29,6 \%)$ este dato puede ser un indicador de la conducta que puede modificar la aceptación de las personas en toma de decisiones en búsqueda de medios para mejorar la salud individual como colectiva.

Nuestro estudio presenta varias limitaciones, tomando en cuenta que las encuestas son reportes instantáneos de la opinión pública y pueden variar de manera dinámica; la encuesta fue enviada vía digital, por lo que la población que no tiene acceso a esta herramienta puede no haber sido representada en la muestra.

Por otro lado, pudo modificar la percepción de las personas, dado que habían estado expuestas recientemente a información relacionada con los debates sobre la vacuna para control de la pandemia.

No contamos con el padrón de la Junta Central Electoral, por lo cual no pudimos realizar un muestreo aleatorio simple, por tal razón acudimos al método no probabilístico y por conveniencia, dando la posibilidad de un sesgo de selección, ya que no se ve demostrado el perfil demográfico nacional en tanto a edad y educación. La posibilidad de sesgo de selección por diseminar el cuestionario en nuestro círculo profesional en la ciudad de Santiago de los Caballeros.

No obstante, más allá de las limitaciones, esperamos que nuestro estudio pueda ayudar a tener una visión más amplia sobre la percepción de la población sobre la vacuna contra el COVID-19, facilitando la creación y dirección de una campaña más efectiva para la vacunación.

\section{Conclusiones}

Tomando en cuenta estos resultados, menos de la mitad de la población de estudio expresó la intención de aplicarse la vacuna, mientras el resto muestra indecisión, lo cual podría ser visto como una oportunidad para aplicar campañas educativas y así aumentar la tasa de aceptación para aplicarla, pudiendo utilizar como eslabones a líderes sociales, representantes de diferentes organizaciones civiles, religiosas, juntas de vecinos, además del sector privado y las autoridades de salud pública. Es útil facilitar diferentes fuentes de acceso, con alcance equitativo a la población para fomentar una información precisa y transparente con un soporte logístico adecuado en todo el territorio nacional.

Recomendamos la implementación de un programa de encuestas semanales concomitantemente con una campańa educativa en los próximos tres meses, la fecha estimada de comienzo la de vacunación contra COVID-19.

\section{Consideraciones Éticas}

Los datos obtenidos en dicho cuestionario fueron utilizados y manejados exclusivamente por el equipo de investigadores para este y posible futuros estudios (fue mencionado en el segmento de consentimiento informado), de forma confidencial sin divulgación de sus identidades. Ninguno de los autores presenta conflicto de intereses.

\section{Agradecimientos}

Agradecemos a los participantes por dedicar su valioso tiempo para responder y diseminar nuestro formulario, aportando en adquisición de los conocimientos necesarios para campañas de vacunación masivas contra el COVID-19 para mitigar los efectos de la pandemia. 


\section{Referencias}

1. Coronavirus (COVID-19) events as they happen [Internet]. [cited 2020 Dec 21]. Available from: https://www.who.int/emergencies/diseases/ novel-coronavirus-2019/events-as-they-happen

2. Home - Johns Hopkins Coronavirus Resource Center [Internet]. [cited 2021 Aug 18]. Available from: https://coronavirus.jhu.edu/

3. COVID-19: Da negativo el italiano que fue primer caso de coronavirus en RD | Hoy Digital [Internet]. [cited 2020 Dec 21]. Available from: https://hoy.com.do/covid-19-da-negativo-el-italiano-que-fue-primer-caso-de-coronavirus-en-rd/

4. Ensheng Dong HDLG. An interactive web-based dashboard to track COVID-19 in real time. the Lancet infectious diseases. 2020 May;20(5):533-4.

5. Yamey G, Schäferhoff M, Hatchett R, Pate M, Zhao F, McDade KK. Ensuring global access to COVID-19 vaccines. The Lancet. 2020 May 2;395(10234):1405-6.

6. Allen A. Vaccine: The Controversial Story of Medicine’s Greatest Lifesaver; 2007.

7. Dubé E, Laberge C, Guay M, Bramadat P, Roy R, Bettinger JA. Vaccine hesitancy: an overview. Human vaccines \& immunotherapeutics. 2013 Aug 8;9(8):1763-73.

8. Kata A. Anti-vaccine activists, Web 2.0, and the postmodern paradigm-An overview of tactics and tropes used online by the anti-vaccination movement. Vaccine. 2012 May 28;30(25):3778-89.

9. Kane MA. Commentary: public perception and the safety of immunization. Vaccine. 1998 Nov;16(Suppl):S73-5.
10. Spier RE. Perception of risk of vaccine adverse events: a historical perspective. Vaccine. 2001 Oct 15;20:S78-84.

11. Streefland P, Chowdhury AM, Ramos-Jimenez P. Patterns of vaccination acceptance. Social science \& medicine. 1999 Dec 1;49(12):1705-16.

12. Poltorak M, Leach M, Fairhead J, Cassell J. 'MMR talk and vaccination choices: An ethnographic study in Brighton. Social Science \& Medicine. 2005 Aug 1;61(3):709-19.

13. Gangel, J. Los expresidentes Obama, Bush y Clinton se ofrecen como voluntarios para vacunarse contra el coronavirus públicamente. $\mathrm{CNN}$. 2020;. Disponible en: https://cnnespanol. cnn.com/2020/12/03/los-expresidentes-obama-bush-y-clinton-se-ofrecen-como-voluntarios-para-vacunarse-contra-el-coronavirus-publicamente/

14. (CDN, 2021) - Presidente Abinader Se pondrá la vacuna Pfizer. CDN. 2021; Disponible en: https://cdn.com.do/nacionales/presidente-luis-abinader-se-pondra-la-vacuna-pfizer/

15. François G, Duclos P, Margolis H, Lavanchy D, Siegrist CA, Meheus A, Lambert PH, Emiroglu N, Badur S, Van Damme P. Vaccine safety controversies and the future of vaccination programs. The Pediatric infectious disease journal. 2005 Nov 1;24(11):953-61.

16. Stefanoff P, Mamelund SE, Robinson M, Netterlid E, Tuells J, Bergsaker MA, Heijbel $\mathrm{H}$, Yarwood J, VACSATC Working Group on Standardization of Attitudinal Studies in Europe. Tracking parental attitudes on vaccination across European countries: the Vaccine Safety, Attitudes, Training and Communication Project (VACSATC). Vaccine. 2010 Aug 9;28(35):5731-7. 
17. Lazarus JV, Ratzan SC, Palayew A, Gostin LO, Larson HJ, Rabin K, Kimball S, El-Mohandes A. A global survey of potential acceptance of a COVID-19 vaccine. Nature medicine. 2020 Oct. 20:1-4.

18. Lobera J, Cabrera P. Percepción social de aspectos científicos del Covid-19. Resultados. 2020; 1-41. Disponible en: https://www.ciencia.gob. es/stfls/MICINN/Ministerio/FICHEROS/ tablas_resultados_encuesta_vacunas_1era_ oleada.pdf

19. Malik AA, McFadden SAM, Elharake J, Omer SB. Determinants of COVID-19 vaccine accep- tance in the US. EClinicalMedicine [Internet]. 2020;26:100495. Available from: https://doi. org/10.1016/j.eclinm.2020.100495

20. Figueiredo A, Simas C, Karafillakis E, Paterson P, Larson HJ. Mapping global trends in vaccine confidence and investigating barriers to vaccine uptake: a large-scale retrospective temporal modelling study. The Lancet. 2020 Sep 26; 396(10255):898-908.

21. Expert Health Care for People of Maryland | University of Maryland Medical System [Internet]. [cited 2021 Jan 23]. Available from: https://www.umms.org.

Tabla 1. Descripción de los participantes y datos de preguntas vacuna COVID-19.

\begin{tabular}{|l|c|}
\hline VARIABLE $(\mathbf{n}=\mathbf{8 4 1})$ & ESTADÍSTICA DESCRIPTIVA \\
\hline Edad (ańos) $($ media $\pm D E)$ & $31.33 \pm 11.22$ \\
\hline Género & $500(59.5)$ \\
\hline Femenino, $n(\%)$ & $341(40.5)$ \\
\hline Masculino, $n(\%)$ & $379(45)$ \\
\hline Mayor nivel educativo alcanzado & $144(17.1)$ \\
\hline Estudiantes Universitario, $n(\%)$ & $149(17.7)$ \\
\hline Licenciatura, $n(\%)$ & $107(12.7)$ \\
\hline Médico, $n(\%)$ & $62(7.5)$ \\
\hline Maestría, $n(\%)$ & \\
\hline Otros, $n(\%)$ & $3(0.4)$ \\
\hline Nacionalidad & $824(98)$ \\
\hline Estadounidense, $n(\%)$ & $2(0.2)$ \\
\hline Dominicano/a, $n(\%)$ & $10(1.2)$ \\
\hline Haitiano/a, $n(\%)$ & \\
\hline Otras, $n(\%)$ & $364(43.4)$ \\
\hline Provincia donde reside en R.D. & $153(18.2)$ \\
\hline Santiago de los Caballeros, $n(\%)$ & $67(8)$ \\
\hline Distrito Nacional, $n(\%)$ & $54(6.4)$ \\
\hline Santo Domingo, $n(\%)$ & $39(4.6)$ \\
\hline Puerto Plata, $n(\%)$ & \\
\hline Espaillat, $n(\%)$ & \\
\hline & \\
\hline
\end{tabular}




\begin{tabular}{|c|c|}
\hline VARIABLE $(n=841)$ & ESTADÍSTICA DESCRIPTIVA \\
\hline Duarte, $n(\%)$ & $30(3.6)$ \\
\hline La Vega, $n(\%)$ & $26(3.1)$ \\
\hline Valverde, $n(\%)$ & $22(2.6)$ \\
\hline San Cristóbal, $n(\%)$ & $14(1.7)$ \\
\hline Santiago Rodríguez, $n(\%)$ & $11(1.3)$ \\
\hline Otras, $n(\%)$ & $61(7.1)$ \\
\hline \multicolumn{2}{|c|}{ Ingresos económicos aproximado mensual RD\$ } \\
\hline Menor de RD \$15,000, $n(\%)$ & $152(18.1)$ \\
\hline Entre RD\$ 15,000 y RD\$20,000, $n(\%)$ & $79(9.4)$ \\
\hline Entre RD\$20,000 y RD\$25,000, $n(\%)$ & $48(5.7)$ \\
\hline Entre RD\$25,000 y RD\$32,000, n (\%) & $53(6.3)$ \\
\hline Entre RD $\$ 32,000$ y RD $\$ 63,000, n(\%)$ & $144(17.1)$ \\
\hline Mayor a $\mathrm{RD} \$ 63,000, n(\%)$ & $157(18.7)$ \\
\hline Sin ingresos económicos, $n(\%)$ & $208(24.7)$ \\
\hline \multicolumn{2}{|l|}{ Profesional de la Salud } \\
\hline No, $n(\%)$ & $552(65.6)$ \\
\hline Sí, $n(\%)$ & $289(34.4)$ \\
\hline \multicolumn{2}{|l|}{ ¿Se aplicará la vacuna contra COVID-19? } \\
\hline No, $n(\%)$ & $143(17)$ \\
\hline No estoy seguro/a, $n(\%)$ & $322(38.3)$ \\
\hline Sí, $n(\%)$ & $376(44.7)$ \\
\hline \multicolumn{2}{|l|}{ ¿Cuándo se aplicaría la vacuna? } \\
\hline En cuanto esté disponible, $n(\%)$ & $242(28.8)$ \\
\hline Esperaría 1-2 meses, $n(\%)$ & $113(13.4)$ \\
\hline Esperaría 6 meses a 1 año, $n(\%)$ & $124(14.7)$ \\
\hline Esperaría de 3-6 meses, $n$ (\%) & $118(14.0)$ \\
\hline Esperaría más de 1 año, $n(\%)$ & $102(12.1)$ \\
\hline \multicolumn{2}{|l|}{ ¿Qué tan efectiva considera será la vacuna? } \\
\hline Nada efectiva, $n(\%)$ & $42(5)$ \\
\hline Poco efectiva, $n(\%)$ & $138(16.4)$ \\
\hline Moderadamente efectiva, $n(\%)$ & $518(61.6)$ \\
\hline Totalmente efectiva, $n(\%)$ & $143(17.0)$ \\
\hline \multicolumn{2}{|l|}{ DE, Desviación estándar } \\
\hline RD, República Dominicana & \\
\hline
\end{tabular}

